\title{
DISTAL BICEPS TENDON RUPTURE - A SINGLE-INCISION REPAIR WITH ENDOBUTTON
}

\author{
Martin Burnev, Georgi Ganchev, Preslav Penev \\ Clinic of Orthopedics and Traumatology, St. Anna Hospital - Varna, \\ Medical University of Varna
}

\begin{abstract}
Distal rupture of the biceps tendon typically occurs in middle aged men during heavy lifting with the elbow flexed at 90 degrees, or when the biceps muscle contracts against unexpected resistance. The estimated incidence is 1.2 per 100,000 population per year. The specific tests for distal biceps tendon ruptures include the hook test and the biceps squeeze test. Acute operative fixation should be offered to all healthy patients who require strength and endurance with elbow flexion and forearm supination. Early anatomic reconstruction of the distal biceps can completely restore elbow flexion and supination strength and endurance and demonstrate higher clinical and functional outcomes. Our preferred technique is a single - incision anatomic repair of the distal biceps tendon using an EndoButton.
\end{abstract}

Keywords: distal biceps tendon rupture, single incision, EndoButton

\section{INTRODUCTION}

Distal biceps tendon ruptures are very rare. They account for only $3 \%$ of all biceps brachii tendon injuries and patients are usually men between the ages of 30 and 60 years(8). The mechanism of injury is nearly always a forceful, eccentric contraction of the biceps muscle. Most injuries are complete ruptures, with the tendon avulsing from the radial tuberosity. Tearing of the bicipital aponeurosis may or may not occur. Classification (7) of distal biceps tendon ruptures is based on chronicity, degree of tear and extent of retraction (Table 1).

Address for correspondence:

Preslav Penev, MD

Clinic of Orthopedics and Traumatology

St. Anna Hospital - Varna

100 Tzar Osvoboditel Blvd.

9000 Varna

e-mail:dr_penev@abv.bg

Received: September 17, 2016

Accepted: September 30, 2016
Table.1. Classification of distal biceps tendon ruptures

Classification of Distal Biceps Tendon Ruptures

PARTIAL RUPTURES

Insertional

Intrasubstance (elongation)

COMPLETE RUPTURES

Acute ( $<4$ weeks)

Chronic ( $>4$ weeks)

Intact aponeurosis

Ruptured aponeurosis

The specific tests for distal biceps tendon ruptures include the hook test (5) and the biceps squeeze test. Surgical reinsertion of the biceps tendon to the radial tuberosity is considered the treatment of choice for most complete ruptures and conservative management leads to deficiency of strength and endurance of forearm supination and elbow flexion. The standard of treatment is a "two-incision" technique, but we use a single, or "one-incision" techniques which utilize only one anterior forearm incision through which the tendon is attached to the radius using a button device. Reported advantages of 


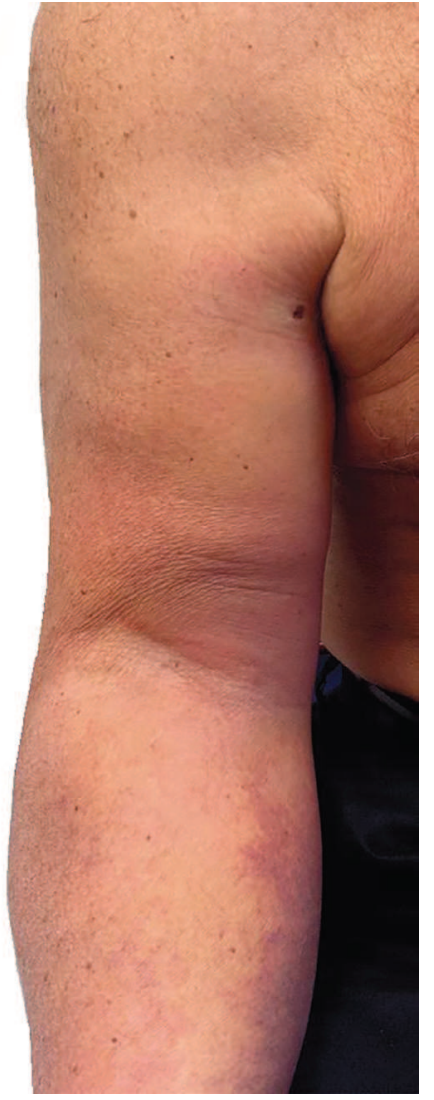

Fig. 1. Deformity and proximal retraction of the biceps, termed a "Popeye muscle"

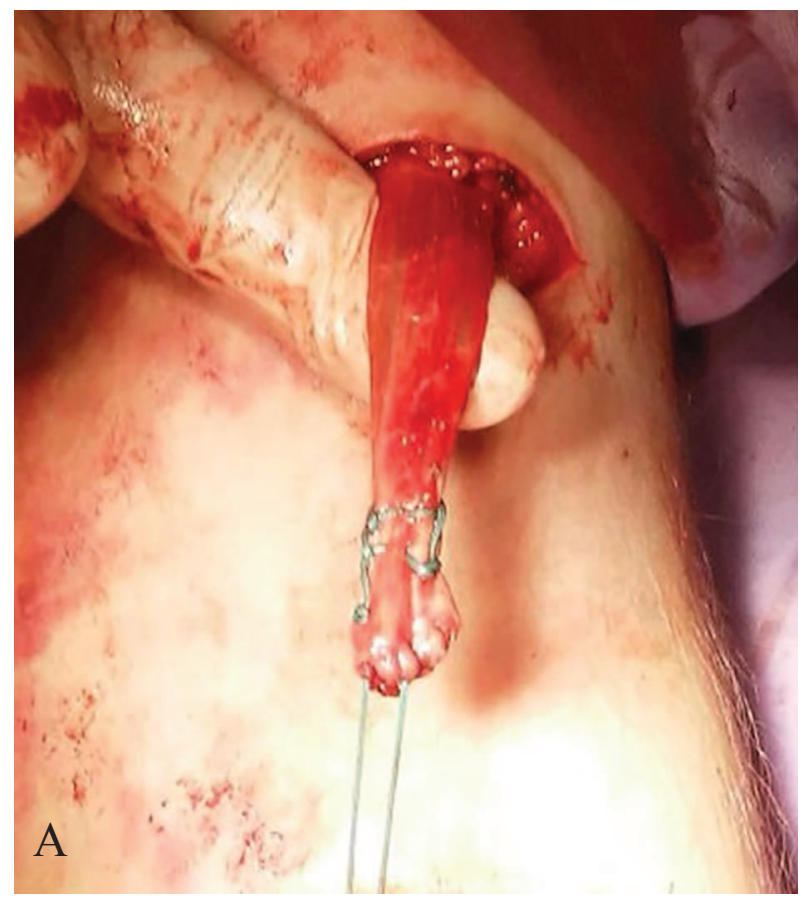

the single-incision technique include limited exposure of the radial tuberosity, better cosmetic result, and decreased risk of heterotopic ossification (2). In general, this method provides adequate fixation strength compared with the intact tendon.

\section{AIM}

The aim of the study is to present the method and results for a single - incision anatomic repair of the distal biceps tendon using an EndoButton.

\section{MATERIALS AND METHODS}

During a period of 24 months (December, 2013 - December, 2015), a total of 17 patients (mean aged $35 \pm 5.2$ ) with acute rupture of the distal biceps tendon were followed. All of them were men and all of them had no congenital deformities and prior injuries. A hook test and a biceps squeeze test were applied and assessed. 15 of the patients were operated on urgently within 48 hours after the injury and two - after the fourth day.

The operative technique consists of a 3 to $4 \mathrm{~cm}$ anterior transverse incision with the arm in extension. Large antecubital veins are ligated or mobilized and the lateral antebrachial cutaneous nerve is identified lateral to the biceps course and carefully pro-

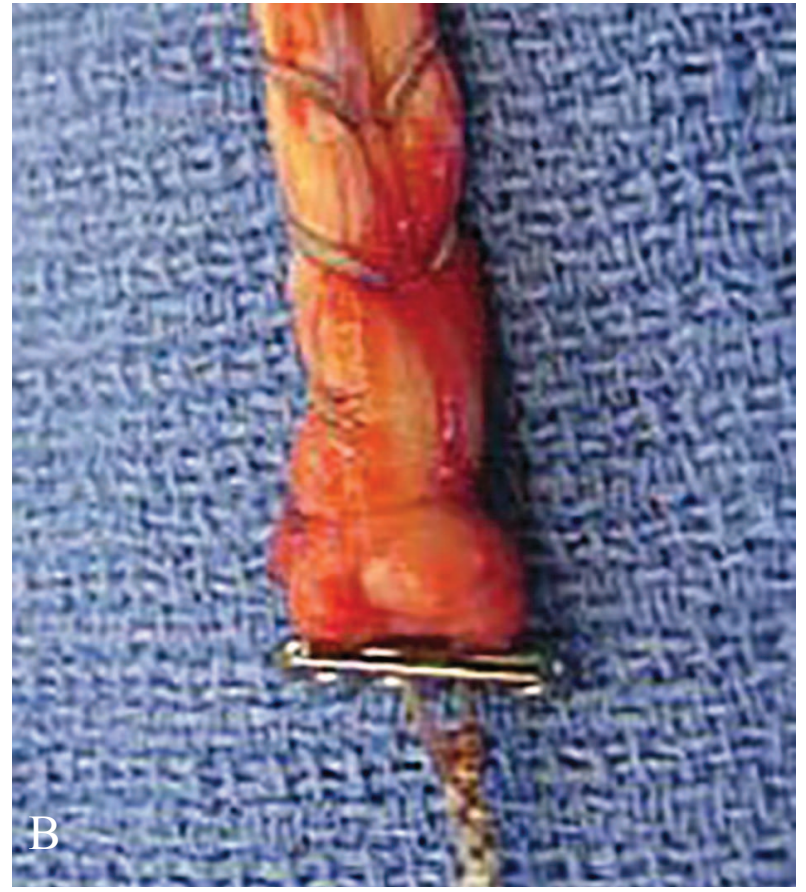

Fig. 2. A-Krakow locking stitches in the tendon end. B - EndoButton attached to the distal end of the biceps tendon 
tected to avoid traction injury. Using a heavy nonabsorbable suture, a whipstitch is woven through the distal biceps tendon from the proximal to the distal part (Figure 2. A).

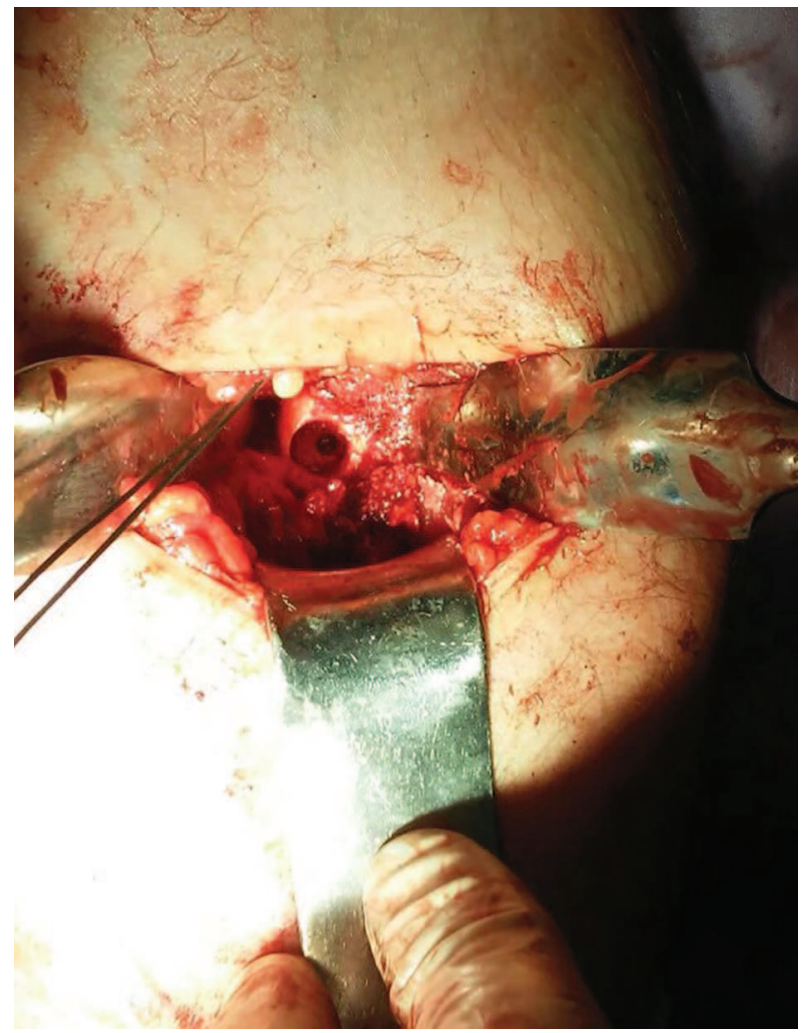

Fig. 3. Tunnel in radial tuberosity

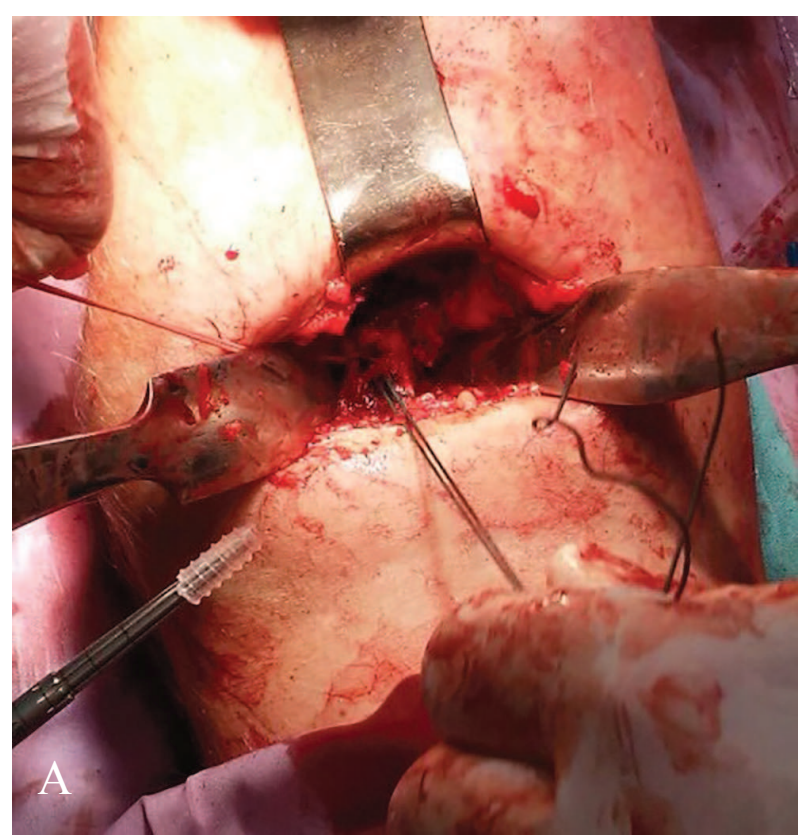

The radial tuberosity is exposed with the elbow in full extension and maximal passive supination to protect the posterior interosseous nerve. A $3.2-\mathrm{mm}$ guide pin is then drilled bicortically through the radial tuberosity. An acorn-shaped reamer is passed over the guide pin to create a unicortical tunnel (Figure 3). The suture limbs are passed through an EndoButton (Figure 2. B), and the EndoButton is passed bicortically through the radial tuberosity (Figure 4A). The tendon is advanced approximately $1 \mathrm{~cm}$ into the tunnel and the sutures are tied, securing the biceps tendon within the radial tuberosity. An interference screw is placed into the tunnel (Figure 4B).

We confirm the correct position of the EndoButton by radiography. The sutures are then pulled through the skin and the closure is routine.

The postoperative care included: posterior splint with the elbow in 90 degrees of flexion and the forearm in neutral rotation for 2 weeks. After that the splint is removed, gentle passive flexion is allowed and full extension is avoided until the sixth week. Active motion for daily activities is allowed in 6 to 12 weeks.

\section{RESULTS}

The results of the repair with EndoButton are very good. Patients' satisfaction was determined as follows: $82 \%$ (14 patients) - excellent, 18\% (3 patients)

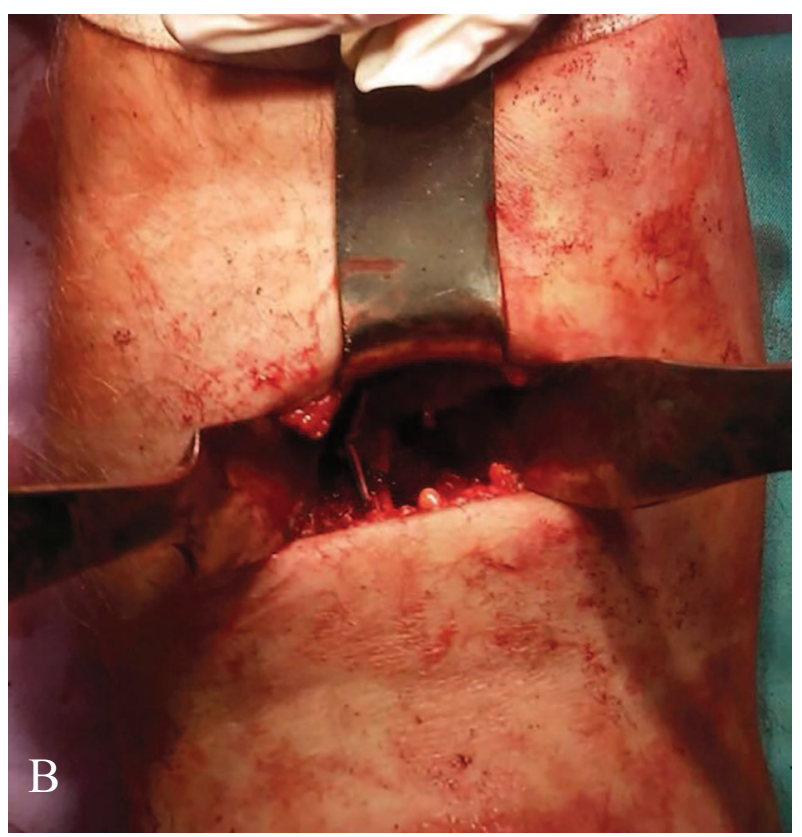

Fig. 4. A - Radial tuberosity with EndoButton suture limbs; B - final result 


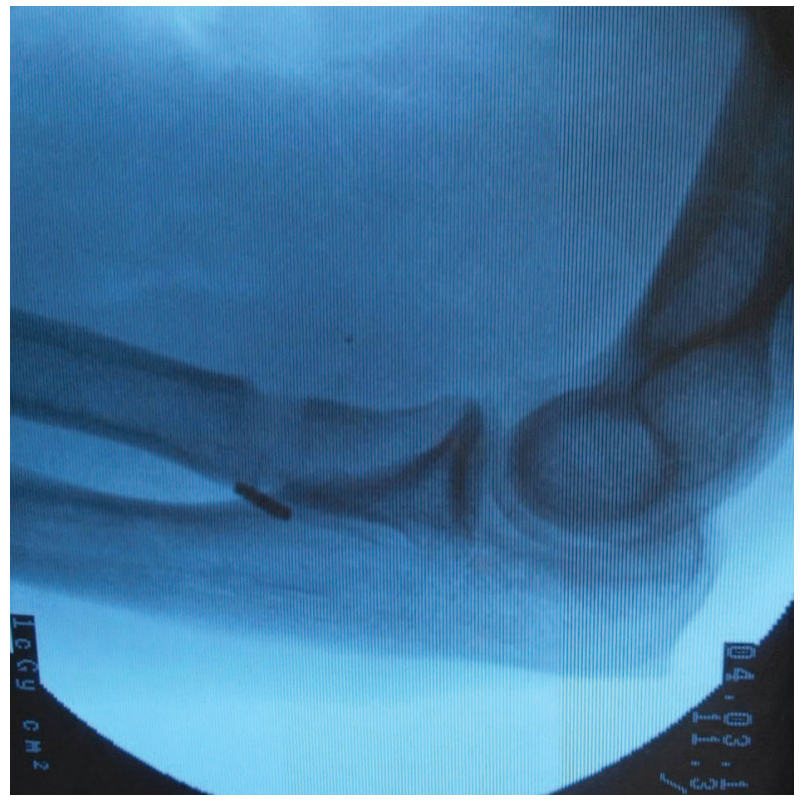

Fig. 5. Proper position confirmed by radiography

- good; there were no results rated as fair or poor. The assessed postoperative Mayo elbow performance score was a total of 96.2 points. Our study has shown virtually $98 \%$ improvement of restoration of flexion and supination strength. Loss of motion is not seen and we have not observed rerupture and superficial radial nerve palsies after EndoButton repair. Clinical studies of other authors with the EndoButton technique also demonstrate similar good results (3).

\section{DISCUSSION}

The reported outcome of surgery for distal biceps tendon rupture is good. Factors that influence the outcome are anatomical reattachment of the tendon and postoperative complications. The largest reported series of distal biceps tendon repairs using a single incision technique found that $96 \%$ were satisfied or very satisfied with the outcome of the surgical repair at an average of 29 months after surgery (4). In a study evaluating outcomes after Endo Button fixation, Peeters et al. (6) reported an average Mayo Elbow Performance Score of 94 points.

There are two major complications associated with this procedure: radial nerve injury and heterotopic ossification. Radial nerve injury has been reported and may be seen as often as in 5\% of the cases after distal biceps tendon reattachment. Rerupture is uncommon. But overall complication rates are similar among the other repair techniques (1).

We intended to use a minimally invasive technique with anterior transverse incision to the best of our abilities. We did not observe complications such as heterotopic ossification or radioulnar synostosis in any of the patients in our series.

\section{CONCLUSION}

The single incision surgical technique used by us with an EndoButton to repair distal biceps tendon rupture is a simple, safe, and effective technique with excellent results and it has been shown to provide the best, most secure anatomical fixation.

\section{REFERENCES}

1. Chavan PR, Duquin TR, Bisson LJ. Repair of the ruptured distal biceps tendon: a systematic review. AmJ Sports Med. 2008;36:1618-1624.

2. Greenberg JA: Endobutton repair of distal biceps tendon ruptures. J Hand Surg Am 34:1541-1548, 2009.

3. Greenberg JA, Femandez JJ, Wang T, et al. EndoButton-assisted repair of distal biceps lZIIdon ruptures. J Shoulder Elbow Surg 2003;12:484-490.

4. McKee MD, Hirji R, Schemitsch EH, et al. Patientoriented functional outcome after repair of distal biceps tendon ruptures using a single-incision technique. J Shoulder Elbow Surg 2005;14(3):302-6.

5. O'Driscoll SW, Goncalves LB, Dietz P: The hook test for distal biceps tendon avulsion. Am J Sports Med 35:1865-1869, 2007.

6. Peeters T, Ching-Soon NG, Jansen N, et al: Functional outcome repair of distal biceps tendon ruptures using the EndoButton technique. J Shoulder Elbow Surg 18:283-287, 2009.

7. Ramsey ML: Distal biceps tendon injuries: diagnosis and management. J Am Acad Orthop Surg 7:199-207, 1999.

8. Safran MR, Graham SM: Distal biceps tendon ruptures: incidence, demographics, and the effect of smoking. Clin Orthop Relat Res 404:275-283, 2002. 